\title{
Resource Active Indicator
}

National Cancer Institute

\section{Source}

National Cancer Institute. Resource Active Indicator. NCI Thesaurus. Code C94066.

Specifies whether the resource is active. 